\title{
Determinants of smoking initiation among women in five European countries: a cross- sectional survey
}

Debora L Oh ${ }^{1,2}$, Julia E Heck ${ }^{1}$, Carolyn Dresler ${ }^{3}$, Shane Allwright ${ }^{4}$, Margaretha Haglund ${ }^{5}$, Sara S Del Mazo ${ }^{6}$, Eva Kralikova ${ }^{7,8}$, Isabelle Stucker ${ }^{9}$, Elizabeth Tamang ${ }^{10}$, Ellen R Gritz ${ }^{11}$, Mia Hashibe ${ }^{1,12^{*}}$

\begin{abstract}
Background: The rate of smoking and lung cancer among women is rising in Europe. The primary aim of this study was to determine why women begin smoking in five different European countries at different stages of the tobacco epidemic and to determine if smoking is associated with certain characteristics and/or beliefs about smoking.

Methods: A cross-sectional telephone survey on knowledge and beliefs about tobacco was conducted as part of the Women in Europe Against Lung Cancer and Smoking (WELAS) Project. A total of 5000 adult women from France, Ireland, Italy, Czech Republic, and Sweden were interviewed, with 1000 from each participating country. All participants were asked questions about demographics, knowledge and beliefs about smoking, and their tobacco use background. Current and former smokers also were asked questions about smoking initiation. Basic statistics on the cross-sectional data was reported with chi-squared and ANOVA p-values. Logistic regression was used to analyze ever versus never smokers. Linear regression analyses were used to analyze age of smoking initiation.

Results: Being older, being divorced, having friends/family who smoke, and having parents who smoke were all significantly associated with ever smoking, though the strength of the associations varied by country. The most frequently reported reason for initiation smoking was friend smoking, with $62.3 \%$ of ever smokers reporting friends as one of the reasons why they began smoking. Mean age of smoking initiation was 18.2 years and over $80 \%$ of participants started smoking by the age of 20. The highest levels of young initiators were in Sweden with 29.3\% of women initiating smoking at age 14-15 and 12.0\% initiating smoking younger than age 14. The lowest level of young initiators was in the Czech Republic with $13.7 \%$ of women initiating smoking at age $14-15$ and $1.4 \%$ of women initiating smoking younger than age 14 . Women who started smoking because their friends smoked or to look 'cool' were more likely to start smoking at a younger age. Women who started smoking to manage stress or to feel less depressed were more likely to start smoking at an older age.
\end{abstract}

Conclusions: In all five participating countries, friends were the primary factor influencing ever smoking, especially among younger women. The majority of participants began smoking in adolescence and the average reported age of smoking initiation was youngest in Sweden and oldest in the Czech Republic.

\footnotetext{
* Correspondence: mia.hashibe@utah.edu

${ }^{1}$ International Agency for Research on Cancer, Lyon, France
} 


\section{Background}

The prevalence of smoking among women varies greatly across European countries, ranging from less than $10 \%$ to over $30 \%$ [1]. Overall, however, Europe has some of the highest levels of female smoking in the world [2]. Furthermore, while smoking among men has declined in some European countries, it is still increasing or stabilised among women in most European countries [3-5].

Reasons for smoking initiation differ across cultures but certain factors have an established association with smoking. Parental, sibling, and friend smoking all have been shown to be strongly associated with an individual's smoking status [6-13]. Low academic achievement as well as low socioeconomic status are also associated with smoking status $[8,12,14-16]$. Women, just like men, begin to smoke in increasing proportions in the population because of marketing [17] and the tobacco industry significantly targets women with focused advertisements and promotions [18]. Women appear to have different reasons for smoking than men; for example, tension reduction and stimulation are thought to be more strongly associated with women smoking than with men $[19,20]$. There is also evidence that weight concerns play a role in the decision to smoke among women [21-24].

Over $60 \%$ of lung cancer cases in European women are attributed to their smoking history $[25,26]$. Thus with smoking rates in women rising, it is not surprising that lung cancer rates in women have also increased in most European countries $[27,28]$. In order to prevent further increases in smoking-related mortality, it is important that anti-tobacco measures target women in Europe.

Many European countries have recently made substantial changes in policy toward smoking and tobacco. The European Community as an entity, in addition to all major European Region countries, except the Czech Republic, has ratified the World Health Organization's Framework Convention on Tobacco Control (FCTC). The FCTC became international law in 2004 and is a comprehensive tobacco control treaty which includes an article for $100 \%$ smoke-free policies. In the same year, Ireland implemented a nation-wide ban on smoking in bars and restaurants. Since then, other European countries, including France, Italy and Sweden, have followed with similar national smoke-free legislation. Other measures against tobacco include increasing taxes on tobacco, improving consumer information, health warning labels on tobacco products, banning tobacco advertising promotion and sponsorship, and facilitating access to smoking cessation treatment [29].

The objective of this study is to determine if ever smoking and smoking initiation are associated with certain characteristics or beliefs about smoking in women in five European countries. In particular, this study aims to elucidate if these characteristics or beliefs about smoking are different for women in countries at different stages in the tobacco epidemic, with northern European countries generally being at a more advanced stage of the epidemic than southern European countries. As current policies are being rapidly reformed in Europe, and as rates of smoking are rising among European women, understanding reasons why European women smoke is crucial in guiding future tobacco control measures.

\section{Methods \\ Participants}

As part of the Women in Europe Against Lung Cancer and Smoking (WELAS) Project, supported by the European Commission, a cross-sectional land-line telephone survey on health attitudes and knowledge was conducted from June to July 2008 in five European countries: France, Ireland, Italy, Czech Republic, and Sweden [30]. These countries were chosen because they are at different stages of the tobacco epidemic and at different stages of enacting tobacco control measures. This study was approved by the International Agency for Research on Cancer ethical review committee.

In total, 5000 women participated in this survey, with 1000 women from each participating country. Of the women reached who were eligible for participation, response rates were $64.8 \%$ in France, $41.4 \%$ in Italy, $59.0 \%$ in Sweden, $54.6 \%$ in Ireland, and $30.6 \%$ in the Czech Republic.

\section{Sampling}

The goal was to have a sample of women from each country that was nationally representative with regard to age and smoking status. Our sampling frame included all adult women 18 years of age and older who had a listed telephone number in the five participating countries. When a household was reached, the woman interviewed was selected at random from all adult women living in that household. To ensure adequate power to examine subsamples of smokers and nonsmokers for each question, smokers were oversampled to reach $28 \%$ of subjects; weighting was done in the analyses to make results representative of each country's female population.

\section{Measures}

The survey was identical across countries, with attention given to translation issues. Surveys were translated by native speakers from each participating country and translations were checked by another native speaker in each country. All participants were asked questions about demographics, beliefs about smoking, and tobacco 
use background in the language native to each country. Current and former smokers were also asked questions about starting smoking.

Our primary outcomes of interest were 1) ever smoking and 2) age at smoking initiation. Women who reported smoking "every day", "some days or occasionally", or "not anymore (>100 cigarettes in life)", were combined to create a group of "ever smokers". Participants with a smoking history were asked at what age they first started smoking regularly.

Background characteristics with prior associations with smoking initiation were included in the analyses, such as age, education, marital status, and income. Since educational systems differ across countries, we measured education as the age at last year of education. Age at last year of education was categorized into less than 16, 16 to 19,20 to 25 , and over 26 years old. These categories were meant to approximately reflect those who did not finish secondary school, those who finished secondary school, those who went to university, and those who had post-graduate education. Respondents reporting being divorced and separated were combined into one category for analysis. In order to create comparable categories between countries, information on personal household income was collected relative to the national median household disposable income for 2005-6 for their respective country. For example, the actual question asked in Ireland was "Given that the median annual household income in Ireland is approximately 43000 euro, would you say that your household income is well below, below, around, above or well above the median?" The national medians provided to respondents were: 30 000 euro for France (2005; source INSEE), 230000 krona for Sweden (2006; source Statistiska centralbyran), 32000 euro for Italy (2006; source Bank of Italy), 110 000 koruna for the Czech Republic (2006; source Czech Statistical Office), 43000 euro for Ireland (2006; source Central Statistics Office Ireland).

Information on friends and family smoking was also collected for the analysis. Those who reported that "more than half" or "most or all" of their friends and family smoked were combined into one category for analyses. Women were also asked if any of their parents, step-parents, or guardians smoked.

Using an open-ended question format, ever smokers were asked to list reasons why they started smoking. Answers were recorded as the participant responded and participants could list more than one reason.

\section{Analyses}

All analyses were done with SAS version 9. Chi-squared tests were conducted to test differences between countries. Logistic regression analyses were conducted to determine factors associated with ever smoking and young smoking initiation. Mean age at initiation was compared across country, age, income category, and education. When comparing age cohorts, those who initiated after the age of $24(\sim 10 \%)$ were excluded to remove age-related bias. One-way ANOVA tests were used to test differences in means. Linear regression was conducted to determine if any reasons for smoking initiation were associated with younger age at initiation.

\section{Results}

A total of 5000 women were interviewed for this study, including 1000 women from each of the five countries. Demographic characteristics are listed in Table 1. Over $40 \%$ of participants $(n=2378)$ had ever smoked $(>100$ cigarettes in lifetime). Age ranged from 18 to 95 with approximately $37 \%$ of participants over the age of 55 (Table 1).

Older age was significantly associated with ever smoking (Table 2). In particular, older women in Italy, the Czech Republic and Sweden all showed a significant increasing trend of a smoking history. Education and income measures were not significantly associated with smoking. In the combined analysis and in country-specific analyses for Ireland, Italy and Sweden, divorce or separation was significantly associated with ever smoking. In the Czech Republic, being widowed was significantly associated with not smoking, as compared to married women (Table 2). The widowed Czech women tended to be older, less educated and have a lower income (data not shown).

Having increasingly more friends and family who smoke was strongly associated with being more likely to smoke. This trend was significant overall and in each country-specific analysis. The associations were especially strong in the Czech Republic and in Sweden. Parent smoking was also significantly associated with ever smoking (Table 2).

In all countries, the primary reason women reported for starting to smoke was because their friends smoked $(62.3 \%)$. The second most frequent reason reported was to make them look 'more cool' (25.5\%). Only $6.1 \%$ of participants reported starting smoking because a family member smoked (Table 3). Some common responses listed under 'Other' were curiosity, rebellion, because other people were smoking, or because they had personal problems.

Average age at smoking initiation was 18.2 and ranged from 10 to 60 years old. Average age at smoking initiation was 17.1 in Sweden, 17.9 in Ireland, 18.1 in France, 18.6 in Italy, and 19.6 in the Czech Republic. Over $80 \%$ of women smokers had started smoking by the age of 20 . Sweden had the highest percentage of women who started smoking at a very young age with $29.3 \%$ starting between 14 and 15 years of age and $12.0 \%$ starting 
Table 1 Women in Europe Against Lung Cancer and Smoking (WELAS) Project demographics by country, weighted.

\begin{tabular}{|c|c|c|c|c|c|c|c|c|}
\hline & \multicolumn{2}{|c|}{$\begin{array}{c}\text { All } \\
(n=5000)\end{array}$} & $\begin{array}{c}\text { France } \\
(\mathrm{n}=1000) \\
\%\end{array}$ & $\begin{array}{c}\text { Ireland } \\
(n=1000) \\
\%\end{array}$ & $\begin{array}{c}\text { Italy } \\
(\mathrm{n}=1000) \\
\%\end{array}$ & $\begin{array}{c}\text { Czech Republic } \\
(\mathrm{n}=1000) \\
\%\end{array}$ & $\begin{array}{c}\text { Sweden } \\
(n=1000) \\
\%\end{array}$ & $\begin{array}{c}\chi^{2} \\
\text { p-value } \\
\text { (df }=4)\end{array}$ \\
\hline \multicolumn{9}{|l|}{ Ever smoker } \\
\hline (>100 cigarettes in life) & 2622 & 41.3 & 37.7 & 49.0 & 39.7 & 34.3 & 45.8 & $<0.0001$ \\
\hline Current smoker & 1421 & 20.5 & 19.9 & 23.5 & 17.5 & 19.2 & 18.6 & 0.0094 \\
\hline \multicolumn{9}{|l|}{ Age } \\
\hline $18-24$ & 426 & 8.5 & 8.2 & 11.4 & 6.5 & 8.3 & 8.2 & $<0.0001$ \\
\hline $25-34$ & 939 & 18.8 & 17.2 & 22.0 & 19.4 & 20.1 & 15.2 & \\
\hline $35-44$ & 916 & 18.3 & 18.6 & 19.8 & 19.0 & 16.0 & 18.2 & \\
\hline $45-54$ & 883 & 17.7 & 18.1 & 16.8 & 16.9 & 17.9 & 18.6 & \\
\hline $55+$ & 1836 & 36.7 & 37.9 & 30.0 & 38.2 & 37.7 & 39.8 & \\
\hline \multicolumn{9}{|l|}{ Age at last education } \\
\hline$<16$ & 601 & 12.5 & 5.0 & 13.2 & 25.2 & 6.0 & 12.3 & $<0.0001$ \\
\hline $16-19$ & 2066 & 42.6 & 37.1 & 52.3 & 34.4 & 49.8 & 39.3 & \\
\hline $20-25$ & 1683 & 35.0 & 53.7 & 29.2 & 30.0 & 35.4 & 27.8 & \\
\hline$>25$ & 476 & 9.7 & 4.0 & 5.3 & 10.4 & 8.9 & 20.6 & \\
\hline (missing) & $(n=174)$ & & $(n=86)$ & $(n=27)$ & $(n=5)$ & $(n=13)$ & $(n=43)$ & \\
\hline \multicolumn{9}{|l|}{ Marital Status } \\
\hline Married & 1979 & 41.1 & 46.3 & 37.1 & 52.1 & 38.0 & 31.9 & $<0.0001$ \\
\hline Divorced/Separated & 727 & 14.2 & 20.5 & 8.2 & 7.8 & 16.9 & 17.5 & \\
\hline Widowed & 605 & 12.8 & 3.5 & 18.2 & 11.6 & 22.1 & 8.9 & \\
\hline Never married & 1074 & 21.6 & 11.2 & 33.0 & 25.4 & 21.6 & 16.6 & \\
\hline Unmarried couple & 514 & 10.4 & 18.5 & 3.6 & 3.1 & 1.4 & 25.1 & \\
\hline (missing) & $(n=101)$ & & $(n=38)$ & $(n=28)$ & $(n=5)$ & $(n=22)$ & $(n=8)$ & \\
\hline \multicolumn{9}{|l|}{ Income } \\
\hline Well below the median & 463 & 9.2 & 7.5 & 7.7 & 17.5 & 8.2 & 5.2 & $<0.0001$ \\
\hline Below the median & 1183 & 23.5 & 24.1 & 25.3 & 29.7 & 19.2 & 19.2 & \\
\hline Around the median & 1386 & 27.6 & 27.4 & 26.5 & 21.6 & 40.8 & 21.7 & \\
\hline Above the median & 805 & 16.0 & 14.9 & 21.9 & 7.4 & 14.5 & 21.2 & \\
\hline Well above the median & 165 & 3.3 & 2.8 & 3.5 & 0.5 & 0.8 & 9.1 & \\
\hline Refused to answer & 998 & 20.4 & 23.3 & 15.1 & 23.31 & 16.5 & 23.6 & \\
\hline \multicolumn{9}{|l|}{ Friends/family smoke } \\
\hline None & 991 & 21.1 & 18.4 & 20.1 & 20.2 & 16.7 & 29.9 & $<0.0001$ \\
\hline Few & 2304 & 47.8 & 42.5 & 52.2 & 44.5 & 45.9 & 53.7 & \\
\hline Less than half & 587 & 11.3 & 13.6 & 10.3 & 10.5 & 13.3 & 8.5 & \\
\hline About half or more & 1094 & 19.9 & 25.5 & 17.4 & 24.8 & 24.1 & 8.0 & \\
\hline (missing) & $(n=24)$ & & $(n=1)$ & $(n=7)$ & $(n=9)$ & $(n=4)$ & $(n=3)$ & \\
\hline \multicolumn{9}{|l|}{ Parents smoke } \\
\hline No & 2101 & 43.1 & 41.4 & 36.2 & 43.2 & 50.9 & 43.8 & $<0.0001$ \\
\hline Yes & 2899 & 56.9 & 58.6 & 63.8 & 56.9 & 49.1 & 56.2 & \\
\hline
\end{tabular}

before the age of 14. The Czech Republic had the lowest percentage of young initiators with $13.7 \%$ starting between the ages of 14 and 15 and $1.4 \%$ starting younger than 14 years old. The highest percentage of women began smoking between the ages of 16 and 17 in all countries, except Sweden (Table 4).

Overall, those who reported currently having lower income had a higher average age of smoking initiation. Average age at initiation did not vary significantly by education in the combined analysis, but did vary significantly in France and Sweden. Women in the younger age cohorts generally began smoking at a younger age than women in older age cohorts (Table 5). Linear regression analysis showed that, after controlling for reasons for starting smoking, age at initiation was significantly earlier among Swedish women $(\beta-0.72, \mathrm{p}=$ $0.02)$ than among French women, and significantly later among women from the Czech Republic ( $\beta$ 1.79, $\mathrm{p}<$ 0.0001) (Table 6). Women who started smoking because their friends smoked or to look 'cool' were more likely 
Table 2 Factors associated with ever smokers compared to never smokers by country, weighted.

\begin{tabular}{|c|c|c|c|c|c|c|}
\hline & $\begin{array}{c}\text { All } \\
(n=5000)\end{array}$ & $\begin{array}{c}\text { France } \\
(n=1000)\end{array}$ & $\begin{array}{c}\text { Ireland } \\
(\mathrm{n}=1000)\end{array}$ & $\begin{array}{c}\text { Italy } \\
(\mathrm{n}=1000)\end{array}$ & $\begin{array}{c}\text { Czech Republic } \\
(n=1000)\end{array}$ & $\begin{array}{c}\text { Sweden } \\
(n=1000)\end{array}$ \\
\hline & OR $(95 \% \mathrm{Cl})$ & OR $(95 \% \mathrm{Cl})$ & OR $(95 \% \mathrm{Cl})$ & OR $(95 \% \mathrm{Cl})$ & OR $(95 \% \mathrm{Cl})$ & OR $(95 \% \mathrm{Cl})$ \\
\hline \multicolumn{7}{|l|}{ Age } \\
\hline $18-24$ & 1.00 & 1.00 & 1.00 & 1.00 & 1.00 & 1.00 \\
\hline $25-34$ & $1.43(1.10,1.86)$ & $2.02(1.08,3.78)$ & $1.66(1.01,2.75)$ & $1.44(0.73,2.83)$ & $0.82(0.44,1.55)$ & $1.56(0.81,3.01)$ \\
\hline $35-44$ & $1.58(1.20,2.08)$ & $1.33(0.71,2.49)$ & $1.81(1.04,3.15)$ & $1.82(0.90,3.67)$ & $1.11(0.54,2.29)$ & $2.50(1.29,4.83)$ \\
\hline $45-54$ & $1.77(1.33,2.34)$ & $1.53(0.83,2.92)$ & $1.70(0.95,3.05)$ & $2.66(1.30,5.45)$ & $1.37(0.66,2.86)$ & $2.57(1.33,4.94)$ \\
\hline $55+$ & $1.68(1.28,2.20)$ & $1.53(0.83,2.79)$ & $1.69(0.94,3.04)$ & $1.98(0.99,3.93)$ & $1.67(0.81,3.46)$ & $2.61(1.37,4.96)$ \\
\hline \multicolumn{7}{|l|}{ Age at last education } \\
\hline$<16$ & $0.98(0.75,1.26)$ & $0.56(0.25,1.26)$ & $1.16(0.57,2.37)$ & $0.89(0.52,1.52)$ & $0.86(0.39,1.90)$ & $0.96(0.59,1.55)$ \\
\hline $16-19$ & $1.09(0.88,1.36)$ & $0.63(0.30,1.33)$ & $1.00(0.54,1.87)$ & $1.13(0.69,1.83)$ & $1.23(0.72,2.08)$ & $1.34(0.92,1.96)$ \\
\hline $20-25$ & $0.85(0.68,1.07)$ & $0.55(0.26,1.13)$ & $0.66(0.35,1.25)$ & $0.96(0.59,1.57)$ & $0.91(0.52,1.58)$ & $0.83(0.56,1.24)$ \\
\hline$>25$ & 1.00 & 1.00 & 1.00 & 1.00 & 1.00 & 1.00 \\
\hline \multicolumn{7}{|l|}{ Marital status } \\
\hline Married & 1.00 & 1.00 & 1.00 & 1.00 & 1.00 & 1.00 \\
\hline Divorced/Separated & $1.49(1.23,1.80)$ & $1.16(0.79,1.67)$ & $1.91(1.12,3.25)$ & $2.00(1.20,3.35)$ & $1.42(0.94,2.16)$ & $1.73(1.14,2.63)$ \\
\hline Widowed & $0.83(0.67,1.04)$ & $1.44(0.67,3.10)$ & $0.77(0.49,1.20)$ & $0.89(0.54,1.46)$ & $0.53(0.33,0.84)$ & $1.36(0.79,2.36)$ \\
\hline Never married & $1.13(0.94,1.35)$ & $1.86(1.14,3.03)$ & $1.28(0.90,1.85)$ & $0.97(0.66,1.41)$ & $1.21(0.75,1.94)$ & $0.89(0.56,1.39)$ \\
\hline Unmarried couple & $1.26(1.01,1.58)$ & $1.22(0.82,1.82)$ & $1.85(0.85,4.02)$ & $1.66(0.76,3.60)$ & $1.64(0.48,5.59)$ & $1.20(0.83,1.73)$ \\
\hline \multicolumn{7}{|l|}{ Income } \\
\hline Well below the median & $1.27(1.00,1.61)$ & $1.57(0.90,2.74)$ & $1.45(0.83,2.54)$ & $1.11(0.71,1.75)$ & $0.73(0.40,1.34)$ & $1.87(0.93,3.75)$ \\
\hline $\begin{array}{l}\text { Below the median } \\
\text { Around the median }\end{array}$ & $\begin{array}{c}1.12(0.94,1.33) \\
1.00\end{array}$ & $\begin{array}{c}1.17(0.79,1.74) \\
1.00\end{array}$ & $\begin{array}{c}1.16(0.80,1.69) \\
1.00\end{array}$ & $\begin{array}{c}0.98(0.67,1.43) \\
1.00\end{array}$ & $\begin{array}{c}1.23(0.81,1.86) \\
1.00\end{array}$ & $\begin{array}{c}1.03(0.67,1.59) \\
1.00\end{array}$ \\
\hline Above the median & $1.17(0.97,1.41)$ & $1.61(1.05,2.49)$ & $1.03(0.69,1.53)$ & $1.00(0.57,1.76)$ & $1.16(0.76,1.79)$ & $1.13(0.75,1.72)$ \\
\hline Well above the median & $0.97(0.68,1.36)$ & $0.85(0.36,2.01)$ & $0.47(0.21,1.04)$ & $2.13(0.23,20.14)$ & $2.14(0.42,10.79)$ & $1.42(0.84,2.43)$ \\
\hline Refused to answer & $0.87(0.72,1.04)$ & $1.14(0.75,1.74)$ & $0.88(0.57,1.35)$ & $0.56(0.37,0.85)$ & $0.81(0.52,1.25)$ & $0.90(0.60,1.34)$ \\
\hline \multicolumn{7}{|l|}{ Friends/family smoke } \\
\hline None & 1.00 & 1.00 & 1.00 & 1.00 & 1.00 & 1.00 \\
\hline Few & $1.45(1.23,1.71)$ & $1.34(0.89,2.02)$ & $1.73(1.20,2.50)$ & $0.97(0.67,1.41)$ & $3.09(1.79,5.33)$ & $1.49(1.09,2.04)$ \\
\hline Less than half & $2.35(1.87,2.95)$ & $1.66(1.00,2.76)$ & $2.25(1.33,3.81)$ & $1.25(0.74,2.09)$ & $8.40(4.48,15.74)$ & $2.69(1.58,4.56)$ \\
\hline About half or more & $3.97(3.22,4.88)$ & $2.57(1.64,4.02)$ & $4.03(2.46,6.63)$ & $3.17(2.08,4.85)$ & $8.26(4.58,14.89)$ & $12.37(5.99,25.55)$ \\
\hline \multicolumn{7}{|l|}{ Parents smoke } \\
\hline No & 1.00 & 1.00 & 1.00 & 1.00 & 1.00 & 1.00 \\
\hline Yes & $1.67(1.47,1.89)$ & $2.21(1.65,2.96)$ & $1.40(1.05,1.87)$ & $1.28(0.97,1.68)$ & $2.21(1.65,2.96)$ & $1.54(1.16,2.04)$ \\
\hline
\end{tabular}

Odds ratios (ever smoker: never smoker) adjusted for all above listed variables (and country for the combined analysis).

to start smoking at a younger age than those who did not cite these reasons. Women who smoked to manage stress or to feel less depressed were more likely to start at an older age (Table 6).

\section{Discussion}

This study provides an overview of factors associated with women's smoking in five European countries at the beginning of a time of rapid change in tobacco policy. We found that friends exert a strong influence on a woman's decision to start smoking in all five countries, especially in younger women. This has been reported from previous studies based in Europe and in studies based in the United States, Iran, Thailand and Mexico [6-12,31-33]. We found that women in the Czech Republic and Sweden appeared to be more strongly influenced by friends and family smoking than in other countries.

We found that being divorced or separated is strongly associated with smoking, which has been reported previously [34]. The association between divorce or separation and ever smoking appears to be strongest in Ireland and Italy, where rates of divorce and separation were lowest in our study sample. Our results also showed that in France, women who had never married were more likely to smoke than those who were married. Chaix and colleagues found similar results when they conducted a nationwide survey in France of 12948 men and women [35].

Overall, we did not find a strong association between socioeconomic measures and smoking. This is in contrast to other findings on education status, low 
Table 3 Reasons reported for smoking initiation among ever smokers, weighted.*

\begin{tabular}{|c|c|c|c|c|c|c|c|}
\hline & $\begin{array}{c}\text { All } \\
(\mathrm{n}=2378) \\
\% \\
\end{array}$ & $\begin{array}{c}\text { France } \\
(n=467) \\
\% \\
\end{array}$ & $\begin{array}{c}\text { Ireland } \\
(\mathrm{n}=519) \\
\% \\
\end{array}$ & $\begin{array}{c}\text { Italy } \\
(\mathrm{n}=474) \\
\%\end{array}$ & $\begin{array}{c}\text { Czech Republic } \\
(n=429) \\
\% \\
\end{array}$ & $\begin{array}{c}\text { Sweden } \\
(\mathrm{n}=489) \\
\% \\
\end{array}$ & $\begin{array}{c}\chi^{2} \\
\text { p-value }\end{array}$ \\
\hline My friends smoked & 62.3 & 67.7 & 65.4 & 58.6 & 61.3 & 58.3 & 0.0152 \\
\hline Smoking made me look more cool & 25.5 & 14.2 & 35.4 & 19.4 & 28.1 & 27.6 & $<0.0001$ \\
\hline My friends approved of my smoking & 6.9 & 5.5 & 16.3 & 0.9 & 10.3 & 0.6 & $<0.0001$ \\
\hline It helped me manage my stress & 6.8 & 8.9 & 8.1 & 7.2 & 7.6 & 2.6 & 0.0021 \\
\hline My family members smoked & 6.1 & 6.1 & 11.1 & 6.4 & 4.6 & 1.7 & $<0.0001$ \\
\hline I was not worried about health effects & 5.9 & 0.4 & 21.1 & 0.2 & 0.9 & 3.0 & $<0.0001$ \\
\hline I believed I could quit whenever I wanted to & 5.6 & 0.7 & 18.2 & 0.2 & 4.1 & 1.8 & $<0.0001$ \\
\hline Tobacco advertising was attractive to me & 1.7 & 1.4 & 4.6 & 0.1 & 0.4 & 1.0 & $<0.0001$ \\
\hline Weight concerns & 1.5 & 1.0 & 5.0 & 0.4 & 0.4 & 0.2 & $<0.0001$ \\
\hline I was less depressed when I smoked & 1.5 & 1.5 & 1.7 & 2.4 & 1.1 & 0.8 & 0.4108 \\
\hline My parents did not mind that I smoked & 0.6 & 0.4 & 0.5 & 0.6 & 0.5 & 1.1 & 0.7187 \\
\hline Other & 15.6 & 11.4 & 14.5 & 24.5 & 13.3 & 14.2 & $<0.0001$ \\
\hline
\end{tabular}

*Participants were allowed to list more than one reason for smoking initiation.

socioeconomic status (SES), and smoking initiation from Europe and other parts of the world [14-16,36-41]. Our null findings could have partly been due the way the data were collected. Instead of asking for what level of education had been achieved, participants were asked how old they were when they completed their education. As individuals can complete different levels of education at different ages, this may have made our education groups more similar in terms of actual education level and biased the results toward the null. Also, since income can be a sensitive topic, there may have been reporting bias. The distribution of income levels was not as we expected; there was a disproportionate number of Italian women who reported having income well below the median and a disproportionate number of Czech women who reported having income around the median. This may be an indication that the way the question was asked made the responses subjective. There was a considerable amount of variation in the response rate between countries. Only $41.4 \%$ of women in Italy and $30.6 \%$ of women in the Czech Republic contacted who were eligible for participation agreed to participate. This may be another reason why the income distribution for these two countries was skewed. Our

Table 4 Distribution of age at smoking initiation by country, weighted.

\begin{tabular}{|c|c|c|c|c|c|c|}
\hline & $\begin{array}{c}\text { All } \\
(n=2364)\end{array}$ & $\begin{array}{c}\text { France } \\
(n=461)\end{array}$ & $\begin{array}{l}\text { Ireland } \\
(n=515)\end{array}$ & $\begin{array}{c}\text { Italy } \\
(\mathrm{n}=472)\end{array}$ & $\begin{array}{l}\text { Czech Republic } \\
\text { (n= 427) }\end{array}$ & $\begin{array}{c}\text { Sweden } \\
(n=489)\end{array}$ \\
\hline Mean age at initiation & 18.2 & 18.1 & 17.9 & 18.6 & 19.6 & 17.1 \\
\hline \multicolumn{7}{|l|}{ (ANOVA p-value < 0.01) } \\
\hline \multicolumn{7}{|c|}{ Range and quartile distribution of age at initiation } \\
\hline Maximum & 60 & 50 & 50 & 55 & 60 & 45 \\
\hline 75\% quartile & 20 & 19 & 20 & 20 & 20 & 18 \\
\hline Median & 17 & 17 & 17 & 18 & 18 & 16 \\
\hline $25 \%$ quartile & 15 & 16 & 15 & 15 & 16 & 14 \\
\hline Minimum & 10 & 11 & 10 & 12 & 10 & 11 \\
\hline \multicolumn{7}{|l|}{ Age at initiation } \\
\hline$<14$ & $6.8 \%$ & $3.2 \%$ & $9.0 \%$ & $6.0 \%$ & $1.4 \%$ & $12.0 \%$ \\
\hline $14-15$ & $20.7 \%$ & $18.1 \%$ & $20.2 \%$ & $19.7 \%$ & $13.7 \%$ & $29.3 \%$ \\
\hline $16-17$ & $24.7 \%$ & $29.6 \%$ & $23.2 \%$ & $22.6 \%$ & $25.9 \%$ & $23.4 \%$ \\
\hline $18-19$ & $20.4 \%$ & $24.7 \%$ & $21.5 \%$ & $20.6 \%$ & $20.6 \%$ & $15.6 \%$ \\
\hline $20-21$ & $13.3 \%$ & $12.7 \%$ & $13.0 \%$ & $14.3 \%$ & $18.2 \%$ & $9.6 \%$ \\
\hline $22-23$ & $4.0 \%$ & $3.4 \%$ & $3.7 \%$ & $5.1 \%$ & $3.9 \%$ & $3.7 \%$ \\
\hline $24-25$ & $3.7 \%$ & $2.6 \%$ & $4.6 \%$ & $4.0 \%$ & $4.8 \%$ & $2.6 \%$ \\
\hline $26-27$ & $1.0 \%$ & $0.8 \%$ & $0.7 \%$ & $1.1 \%$ & $1.9 \%$ & $0.9 \%$ \\
\hline $28-29$ & $0.8 \%$ & $0.7 \%$ & $1.0 \%$ & $0.4 \%$ & $0.9 \%$ & $1.0 \%$ \\
\hline $30+$ & $4.6 \%$ & $4.2 \%$ & $3.1 \%$ & $6.2 \%$ & $8.7 \%$ & $2.0 \%$ \\
\hline
\end{tabular}


Table 5 Mean age at smoking initiation by income, education, and age, weighted.

\begin{tabular}{|c|c|c|c|c|c|c|c|c|c|c|c|c|}
\hline \multirow[b]{2}{*}{ Mean age at initiation } & \multicolumn{2}{|c|}{$\begin{array}{c}\text { All } \\
(n=2364) \\
\end{array}$} & \multicolumn{2}{|c|}{$\begin{array}{c}\text { France } \\
(n=461)\end{array}$} & \multicolumn{2}{|c|}{$\begin{array}{l}\text { Ireland } \\
(\mathrm{n}=515)\end{array}$} & \multicolumn{2}{|c|}{$\begin{array}{c}\text { Italy } \\
(n=472)\end{array}$} & \multicolumn{2}{|c|}{$\begin{array}{l}\text { Czech Republic } \\
(n=427)\end{array}$} & \multicolumn{2}{|c|}{$\begin{array}{l}\text { Sweden } \\
(n=489)\end{array}$} \\
\hline & $\mathrm{n}$ & mean & $\mathrm{n}$ & mean & $\mathrm{n}$ & mean & $\mathrm{n}$ & mean & $\mathrm{n}$ & mean & $\mathrm{n}$ & mean \\
\hline \multicolumn{13}{|l|}{ by current income } \\
\hline Well below the median & 241 & 19.3 & 49 & 18.5 & 41 & 20.3 & 88 & 18.7 & 28 & 20.9 & 35 & 19.1 \\
\hline Below the median & 591 & 18.3 & 114 & 17.8 & 144 & 17.9 & 143 & 18.3 & 92 & 21.1 & 98 & 17.0 \\
\hline Around the median & 647 & 18.1 & 111 & 18.4 & 144 & 17.6 & 113 & 18.3 & 175 & 18.9 & 104 & 17.0 \\
\hline Above the median & 400 & 17.7 & 79 & 18.4 & 107 & 17.4 & 41 & 18.6 & 71 & 18.7 & 102 & 16.6 \\
\hline Well above the median & 79 & 17.9 & 14 & 20.0 & 11 & 19.6 & 4 & 22.8 & 4 & 17.3 & 46 & 16.6 \\
\hline Refused to answer & 406 & 18.1 & 94 & 17.3 & 68 & 17.8 & 83 & 19.3 & 57 & 20.2 & 104 & 17.0 \\
\hline (ANOVA p-value) & \multicolumn{2}{|c|}{$(p=0.0025)$} & \multicolumn{2}{|c|}{$(p=0.0945)$} & \multicolumn{2}{|c|}{$(p=0.0101)$} & \multicolumn{2}{|c|}{$(p=0.4711)$} & \multicolumn{2}{|c|}{$(p=0.0077)$} & \multicolumn{2}{|c|}{$(p=0.0566)$} \\
\hline \multicolumn{13}{|l|}{ by age at last education } \\
\hline$<16$ & 288 & 18.8 & 21 & 20.9 & 89 & 17.9 & 104 & 19.7 & 19 & 21.2 & 55 & 17.3 \\
\hline $16-19$ & 1052 & 18.0 & 165 & 18.2 & 278 & 18.0 & 175 & 18.1 & 228 & 19.7 & 206 & 16.3 \\
\hline $20-25$ & 713 & 18.2 & 214 & 18.0 & 112 & 17.8 & 140 & 18.3 & 138 & 19.1 & 109 & 17.7 \\
\hline$>25$ & 229 & 18.3 & 19 & 17.6 & 30 & 18.3 & 50 & 18.8 & 35 & 20.3 & 95 & 17.6 \\
\hline Missing & 82 & 17.3 & 42 & 17.2 & 6 & 14.3 & 3 & 19.4 & 7 & 18.9 & 24 & 17.5 \\
\hline (ANOVA p-value) & \multicolumn{2}{|c|}{$(p=0.0913)$} & \multicolumn{2}{|c|}{$(p=0.0162)$} & \multicolumn{2}{|c|}{$(p=0.9321)$} & \multicolumn{2}{|c|}{$(p=0.0807)$} & \multicolumn{2}{|c|}{$(p=0.3701)$} & \multicolumn{2}{|c|}{$(p=0.0071)$} \\
\hline \multicolumn{13}{|l|}{ by current age* } \\
\hline $18-24$ & 184 & & 30 & & 50 & & 30 & & 37 & & 37 & \\
\hline $25-34$ & 419 & 16.4 & 76 & 16.9 & 110 & 16.4 & 92 & 16.3 & 80 & 17.2 & 61 & 15.2 \\
\hline $35-44$ & 380 & 17.0 & 61 & 17.2 & 102 & 17.2 & 74 & 17.1 & 52 & 17.8 & 91 & 16.2 \\
\hline $45-54$ & 391 & 17.0 & 74 & 16.8 & 78 & 17.0 & 87 & 17.3 & 60 & 17.9 & 92 & 16.2 \\
\hline $55+$ & 743 & 17.6 & 179 & 17.7 & 129 & 17.6 & 136 & 17.9 & 122 & 18.5 & 177 & 16.9 \\
\hline (ANOVA p-value) & \multicolumn{2}{|c|}{$(p<0.0001)$} & \multicolumn{2}{|c|}{$(p=0.0002)$} & \multicolumn{2}{|c|}{$(p<0.0001)$} & \multicolumn{2}{|c|}{$(p<0.0001)$} & \multicolumn{2}{|c|}{$(p<0.0001)$} & \multicolumn{2}{|c|}{$(p<0.0001)$} \\
\hline
\end{tabular}

*excluding those who initiated after the age of $24(n=245)$

Table 6 Linear regression of factors associated with age at smoking initiation.

\begin{tabular}{lcc}
\hline & $\boldsymbol{\beta}(\mathbf{9 5 \%} \mathrm{Cl})$ & $\mathbf{p}$-value \\
\hline Intercept & $18.74(18.20,19.28)$ & $<0.0001$ \\
Country & - & 0.47 \\
France & $0.22(-0.38,0.82)$ & 0.08 \\
Ireland & $0.54(-0.06,1.14)$ & $<0.0001$ \\
Italy & $1.79(1.16,2.41)$ & 0.02 \\
Czech & $-0.72(-1.30,-0.14)$ & $<0.0001$ \\
Sweden & & $<0.0001$ \\
Reasons for initiation* & $-1.02(-1.42,-0.61)$ & 0.35 \\
My friends smoked & $-1.69(-2.15,-1.23)$ & $<0.0001$ \\
Smoking made me look more cool & $-0.36(-1.11,0.39)$ & 0.61 \\
My friends approved of my smoking & $2.67(1.88,3.45)$ & 0.44 \\
It helped me manage my stress & $-0.20(-0.98,0.58)$ & 0.22 \\
My family members smoked & $0.41(-0.63,1.45)$ & 0.37 \\
I was not worried about health effects & $-0.65(-1.69,0.39)$ & 0.56 \\
I believed I could quit whenever I wanted to & $0.67(-0.79,2.14)$ & $<0.0001$ \\
Tobacco advertising was attractive to me & $0.45(-1.08,1.99)$ & 0.42 \\
Weight concerns & $3.88(2.33,5.42)$ & $-0.95(-3.27,1.37)$ \\
I was less depressed when I smoked & & \\
My parents did not mind that I smoked & & \\
\hline
\end{tabular}

*Respondents could choose more than one reason for initiation. Those who gave each reason were compared with those who did not. 
null findings on socio-economic status and smoking initiation could have also been due to generational differences between the younger and older women. In younger women, less education has been found to be associated with ever smoking; however, education has been found to be less predictive for older age groups. The generational shift varies in different European countries, with larger inequalities in Northern Europe, and this could have also diluted our overall results [42-47].

Alternatively, the null association between socioeconomic measures and ever smoking could in fact be accurate. Most studies investigating smoking initiation and socioeconomic factors look at populations that include both men and women. Women, however, may be less influenced by education and income status than men and more influenced by social factors such as friends and family. Many studies looking at SES and smoking status measure SES at the neighborhood level $[14,15,36,38]$. Using neighborhood level data, rather than individual level data, may account for societal factors that may have a strong influence on women. One study showed that higher levels of smoking in deprived areas can only be partially explained by individual SES [15]. Similarly, a study based in California found that the protective effects of individual SES may be reduced if an individual lives in a low SES neighborhood [36]. Further investigation may provide interesting insight into what specific aspects of SES influence smoking in women.

As has been seen elsewhere [6-12], friends smoking was the top reason given for smoking initiation. The second most common reason for initiation was to look cool'. Spijkerman and colleagues also found that smoking was associated with wanting to be cool, rebellious and attractive [48]. Watson and colleagues found that smoking was accepted as part of a 'cool' image [49]. Friends smoking and looking cool was especially important for the younger women in our study.

Over $80 \%$ of the smokers in our study started smoking by 20 years of age. Those who initiate smoking in adolescence are of particular concern. Adolescents are more susceptible to nicotine addiction and require a shorter duration of smoking and fewer cigarettes to become addicted [50]. Furthermore, the earlier one starts smoking, the greater the cancer risk in middle and old age $[50,51]$. Average age at smoking initiation varied by country, income, education, and age cohort. Schulze and Mons also found mean age of smoking initiation differed by education level in Germany, and they found that the gap was widening among women [52]. Consistent with our findings, La Vecchia et al. found that Italian women are initiating smoking at an earlier age than previous generations [53]. Differences in age at initiation by country may be reflected in inter-country variation in lung cancer mortality by age [3].

We found depression to be associated with later smoking initiation. Since median age of onset of mood disorders, including major depressive disorder, ranges from 29 to 43 globally [54], it is not surprising that women who initiated smoking at a later age cited depression as a factor. In our study sample, $7.3 \%$ of women who started smoking at age 29 and older cited depression as a reason for initiating smoking compared to $1.2 \%$ in women who started smoking younger than 29 ( $\mathrm{p}<0.0001$, data not shown). There is already much evidence linking depression and smoking $[55,56]$; however, there is some debate as to the direction of the causality [57-61].

However, since depression was only evaluated by selfreport, the definition of depression may have been used very broadly by the participants and it is difficult to make any specific clinical conclusions. It may be interesting for future studies to investigate if the relationship between depression and smoking differs by age at smoking initiation.

We found that individuals who stated they began smoking to reduce stress were more likely to have initiated at an older age. Other studies have found an association between stress and smoking [62-64]. Lloyd and Taylor found that the effect of former life stress predicts smoking independently of recent stress and that the effect of social stress on smoking is additive over time [65]. In other words, stress at a younger age may affect future smoking; therefore, women who initiated smoking at a later age were more likely to cite stress as a factor.

One major limitation of the study was the stratified sampling approach using available telephone numbers. This method was chosen to allow the study to reach a large sample of women that was proportionally representative of smoking rates in each age group. However, administering the survey via telephone prevented us from verifying self-reported data. The potential for recall bias on reported age of initiation may have affected the accuracy of results. This bias was not likely to be differential by age group and thus should not affect the overall picture of the results. Also, since no mobile phone numbers were included in the phone list, the study could have excluded a substantial number of women from our study who may have unknown differences than those who could be reached by home telephone. Level of income and/or education may have affected whether women had a landline or whether they relied solely on cellular phone use. Thus, the participants may not be an accurate representation of their respective countries. 
Though the five countries included in our study were at different stages of the tobacco epidemic [27] factors associated with smoking initiation were very similar. The association between friends and family smoking was found in all countries, but was strongest in Sweden and Czech Republic, two countries with very different tobacco epidemics. Mean age of smoking initiation was youngest in Sweden, which is in the more advanced stages of the tobacco epidemic. Mean age of initiation was oldest in the Czech Republic, which is in an earlier stage of the tobacco epidemic.

As rates of smoking are still rising among women in much of Europe, there is much to be done to prevent smoking-attributable mortality rates from also rising. The recent changes in Europe's tobacco policy have shown promising signs of slowing the epidemic. In a cross-sectional study of 18 European countries, countries with more developed tobacco control policies had higher levels of smoking cessation than in countries with less developed tobacco policies [66]. In Ireland, Mullally et al. found a significant decline in cigarette consumption among bar workers and a significant drop in smoking prevalence in the general population one year after a nationwide ban on workplace smoking in March 2004 [67]. In Scotland, Fowkes et al. found that smoking quit rates increased in the three months prior to introduction of the Scottish smoke-free legislation in March 2006 [68].

\section{Conclusions}

This study showed that among European women, friends are the primary factor influencing both ever smoking and smoking initiation, especially among young women. We also found that over $80 \%$ of European women begin smoking by the age of 20 and that average age of smoking initiation varies by country, income, and age cohort, with Swedish women most likely to start smoking at a young age. The results from this study may serve as a helpful baseline for future studies on the long-term effect of the recent European tobacco laws.

\footnotetext{
Acknowledgements

This publication arises from the project Women in Europe Against Lung Cancer and Smoking (WELAS) which has received funding from the European Union, in the framework of the Public Health Programme. Debora $\mathrm{L}$ Oh worked on this project under the tenure of a special training award from the International Agency for Research on Cancer.
}

\section{Author details}

'International Agency for Research on Cancer, Lyon, France. ${ }^{2}$ Department of Epidemiology, University of California Los Angeles, School of Public Health, Los Angeles, California, USA. ${ }^{3}$ Tobacco Prevention and Cessation Program, Arkansas Department of Health, Little Rock, Arkansas, USA. ${ }^{4}$ Department of Public Health and Primary Care, Trinity College Dublin, Republic of Ireland. ${ }^{5}$ Tobacco Control, National Institute of Public Health, Östersund, Sweden. ${ }^{6}$ Health Professionals Against Tobacco, Stockholm, Sweden. ${ }^{7}$ Institute of Hygiene and Epidemiology, First Faculty of Medicine, Charles University and
General University Hospital, Prague, Czech Republic. ${ }^{8} 3$ rd Medical Department - Department of Endocrinology and Metabolism, First Faculty of Medicine, Charles University and General University Hospital, Prague, Czech Republic. ${ }^{9}$ INSERM U 754 - IFR69, Villejuif, France. ${ }^{10}$ Regione del Veneto Direzione Prevenzione, Servizio di Sanità Pubblica e Screening, Venezia, Italy. ${ }^{11}$ Department of Behavioral Science, The University of Texas MD Anderson Cancer Center, Houston, Texas, USA. ${ }^{12}$ Division of Public Health, Department of Family \& Preventive Medicine, University of Utah School of Medicine, Salt Lake City, Utah, USA.

\section{Authors' contributions}

DLO analyzed the data and drafted the manuscript. JEH assisted in the design of the study and the statistical analysis. CD, SA, M Haglund, SSDM, EK, IS, ET, and ERG helped to draft the manuscript. M Hashibe conceived of the study, and participated in its design and coordination and helped to draft the manuscript. All authors read and approved the final manuscript.

\section{Competing interests}

This research was funded by the European Union, in the framework of the Public Health Programme.

Received: 12 October 2009

Accepted: 17 February 2010 Published: 17 February 2010

\section{References}

1. WHO. Regional Office for Europe: Tobacco control database. WHO 2008.

2. Warren CW, Jones NR, Eriksen MP, Asma S: Patterns of global tobacco use in young people and implications for future chronic disease burden in adults. The Lancet 2006, 367:749-753.

3. Bosetti C, Levi F, Lucchini F, Negri E, Vecchia CL: Lung cancer mortality in European women: recent trends and perspectives. Annals of Oncology 2005, 16:1597-1604.

4. Hublet A, De Bacquer D, Valimaa R, Godeau E, Schmid H, Rahav G, Maes L: Smoking trends among adolescents from 1990 to 2002 in ten European countries and Canada. BMC Public Health 2006, 6:280.

5. Zatonski WA, Manczuk M, Powles J, Negri E: Convergence of male and female lung cancer mortality at younger ages in the European Union and Russia. The European Journal of Public Health 2007, 17:450-454.

6. Carvajal SC, Wiatrek DE, Evans RI, Knee CR, Nash SG: Psychosocial determinants of the onset and escalation of smoking: cross-sectional and prospective findings in multiethnic middle school samples. Journal of Adolescent Health 2000, 27:255-265.

7. de Vries H, Engels R, Kremers S, Wetzels J, Mudde A: Parents' and friends' smoking status as predictors of smoking onset: findings from six European countries. Health Education Research 2003, 18:627-636.

8. Forrester K, Biglan A, Severson HH, Smolkowski K: Predictors of smoking onset over two years. Nicotine \& Tobacco Research 2007, 9:1259-1267.

9. Kokkevi A, Richardson C, Florescu S, Kuzman M, Stergar E: Psychosocial correlates of substance use in adolescence: A cross-national study in six European countries. Drug and Alcohol Dependence 2007, 86:67-74.

10. Kokkevi AE, Arapaki AA, Richardson C, Florescu S, Kuzman M, Stergar E: Further investigation of psychological and environmental correlates of substance use in adolescence in six European countries. Drug and Alcohol Dependence 2007, 88:308-312.

11. Nichols TR, Birnbaum AS, Birnel S, Botvin GJ: Perceived smoking environment and smoking initiation among multi-ethnic urban girls. Journal of Adolescent Health 2006, 38:369-375.

12. Wilkinson A, Schabath M, Prokhorov A, Spitz M: Age-related differences in factors associated with smoking initiation. Cancer Causes and Control 2007, 18:635-644.

13. Gritz ER, Prokhorov AV, Hudmon KS, Jones MM, Rosenblum C, Chung-Chi C, Chamberlain RM, Taylor WC, Johnston D, de Moor C: Predictors of susceptibility to smoking and ever smoking: A longitudinal study in a triethnic sample of adolescents. Nicotine \& Tobacco Research 2003, 5:493.

14. Dragano N, Bobak M, Wege N, Peasey A, Verde P, Kubinova R, Weyers S, Moebus S, Mohlenkamp S, Stang A, Erbel R, Jockel KH, Siegrist J, Pikhart H: Neighbourhood socioeconomic status and cardiovascular risk factors: a multilevel analysis of nine cities in the Czech Republic and Germany. BMC Public Health 2007, 7:255. 
15. Reijneveld SA: The impact of individual and area characteristics on urban socioeconomic differences in health and smoking. Int J Epidemiol 1998, 27:33-40.

16. Willemsen MC, Hoogenveen RT, Lucht van der F: New smokers and quitters: Transitions in smoking status in a national population. The European Journal of Public Health 2002, 12:136-138

17. DiFranza JR, Wellman RJ, Sargent JD, Weitzman M, Hipple BJ, Winickoff JP, for the Tobacco Consortium CfCHRotAAoP: Tobacco Promotion and the Initiation of Tobacco Use: Assessing the Evidence for Causality. Pediatrics 2006, 117:e1237-1248.

18. Barbeau EM, Leavy-Sperounis A, Balbach ED: Smoking, social class, and gender: what can public health learn from the tobacco industry about disparities in smoking?. Tob Control 2004, 13:115-120.

19. Berlin I, Singleton EG, Pedarriosse A, Lancrenon S, Rames A, Aubin H, Niaura R: The Modified Reasons for Smoking Scale: factorial structure, gender effects and relationship with nicotine dependence and smoking cessation in French smokers. Addiction 2003, 98:1575-1583.

20. Rowe K, Macleod Clark J: Why nurses smoke: a review of the literature. International Journal of Nursing Studies 2000, 37:173-181.

21. Honjo K, Siegel M: Perceived importance of being thin and smoking initiation among young girls. Tobacco Control 2003, 12:289-295.

22. French $S A$, Perry $C L$, Leon $G R$, Fulkerson JA: Weight concerns, dieting behavior, and smoking initiation among adolescents: a prospective study. American Journal of Public Health 1994, 84:1818-1820.

23. Kaufman AR, Augustson EM: Predictors of regular cigarette smoking among adolescent females: Does body image matter?. Nicotine \& Tobacco Research 2008, 10:1301-1309.

24. Weiss JW, Merrill V, Gritz ER: Ethnic variation in the association between weight concern and adolescent smoking. Addictive Behaviors 2007, 32:2311-2316.

25. Simonato L, Agudo A, Ahrens W, Benhamou E, Benhamou S, Bofetta P, Brennan P, Darby SC, Forastiere F, et al: Lung cancer and cigarette smoking in Europe: An update of risk estimates and an assessment of inter-country heterogeneity. International Journal of Cancer 2001, 91:876-887.

26. Peto R, Alan DLopez, Jillian Boreham, Thun M: Mortality from smoking in developed countries 1950-2000 (European Union - 25 countries). 2006http://www.ctsu.ox.ac.uk/ tobacco/SMK_All_PAGES.pdf.

27. Bray F, Tyczynski JE, Parkin DM: Going up or coming down? The changing phases of the lung cancer epidemic from 1967 to 1999 in the 15 European Union countries. European Journal of Cancer 2004, 40:96-125.

28. Levi F, Bosetti C, Fernandez F, Hill C, Lucchini F, Negri E, La Vecchia C: Trends in lung cancer among young European women: The rising epidemic in France and Spain. International Journal of Cancer 2007. 121:462-465.

29. Joossens L, Raw M: The Tobacco Control Scale: a new scale to measure country activity. Tobacco Control 2006, 15:247-253.

30. Heck JE, Stucker I, Allwright S, Gritz ER, Haglund M, Healton CG, Kralikova E, Sanchez Del Mazo S, Tamang E, Dresler CM, Hashibe M: Home and workplace smoking bans in Italy, Ireland, Sweden, France, and the Czech Republic. Eur Respir J

31. Kelishadi R, Mokhtari M, Tavasoli A, Khosravi A, hangar-Nazari I, Sabet B, Kazemi A, Amini A: Determinants of tobacco use among youths in Isfahan, Iran. International Journal of Public Health 2007, 52:173-179.

32. Nuno-Gutierrez BL, Alvarez-Nemegyei J, Madrigal-De Leon EA, TapiaCuriel A: Factores asociados a los patrones de consumo de tabaco en adolescentes escolares. (Spanish). Revista Medica del IMSS 2008, 46:19-26.

33. Rudatsikira E, Muula A, Siziya S, Mataya R: Correlates of cigarette smoking among school-going adolescents in Thailand: findings from the Thai global youth tobacco survey 2005. International Archives of Medicine 2008, $1: 8$

34. Osler M, McGue M, Lund R, Christensen K: Marital Status and Twins' Health and Behavior: An Analysis of Middle-Aged Danish Twins. Psychosomatic Medicine 2008, 70:482-487.

35. Chaix B, Guilbert P, Chauvin P: A multilevel analysis of tobacco use and tobacco consumption levels in France. Are there any combination risk groups?. The European Journal of Public Health 2004, 14:186-190.

36. Chuang YC, Cubbin C, Ahn D, Winkleby MA: Effects of neighbourhood socioeconomic status and convenience store concentration on individual level smoking. Journal of Epidemiology and Community Health 2005, 59:568-573.
37. Mathur C, Stigler MH, Perry CL, Arora M, Reddy KS: Differences in prevalence of tobacco use among Indian urban youth: The role of socioeconomic status. Nicotine \& Tobacco Research 2008, 10:109-116.

38. Najman JM, Toloo G, Siskind V: Socioeconomic disadvantage and changes in health risk behaviours in Australia: 1989-90 to 2001. Bulletin of the World Health Organization 2006, 84:976-984.

39. Watt HC, Carson C, Lawlor DA, Patel R, Ebrahim S: Influence of Life Course Socioeconomic Position on Older Women's Health Behaviors: Findings From the British Women's Heart and Health Study. Am J Public Health 2009, 99:320-327.

40. Unger JB, Sun $P$, Johnson CA: Socioeconomic correlates of smoking among an ethnically diverse sample of 8 th grade adolescents in Southern California. Preventive Medicine 2007, 44:323-327.

41. Moussa KM, Ostergren PO, Grahn M, Kunst AE, Eek F, Essen B: Socioeconomic differences in smoking trends among pregnant women at first antenatal visit in Sweden 1982-2001: Increasing importance of educational level for the total burden of smoking. Tob Control 2008, tc. 2008.026336.

42. Huisman M, Kunst AE, Mackenbach JP: Inequalities in the prevalence of smoking in the European Union: comparing education and income. Prev Med 2005, 40:756-764.

43. Huisman M, Kunst AE, Mackenbach JP: Educational inequalities in smoking among men and women aged 16 years and older in 11 European countries. Tob Control 2005, 14:106-113.

44. Schaap MM, Kunst AE: Monitoring of socio-economic inequalities in smoking: learning from the experiences of recent scientific studies. Public Health 2009, 123:103-109.

45. Schaap MM, van Agt HM, Kunst AE: Identification of socioeconomic groups at increased risk for smoking in European countries: looking beyond educational level. Nicotine Tob Res 2008, 10:359-369.

46. Giskes K, Kunst AE, Benach J, Borrell C, Costa G, Dahl E, Dalstra JA, Federico B, Helmert U, Judge K, Lahelma E, Moussa K, Ostergren PO, Platt S, Prattala R, Rasmussen NK, Mackenbach JP: Trends in smoking behaviour between 1985 and 2000 in nine European countries by education. $J$ Epidemiol Community Health 2005, 59:395-401.

47. Cavelaars AE, Kunst AE, Geurts JJ, Crialesi R, Grotvedt L, Helmert U, Lahelma E, Lundberg O, Matheson J, Mielck A, Rasmussen NK, Regidor E, do Rosario-Giraldes M, Spuhler T, Mackenbach JP: Educational differences in smoking: international comparison. BMJ 2000, 320:1102-1107.

48. Spijkerman R, Eijnden van den RJJM, Engels RCME: Self-comparison processes, prototypes, and smoking onset among early adolescents. Preventive Medicine 2005, 40:785-794.

49. Watson NA, Clarkson JP, Donovan RJ, Giles-Corti B: Filthy or fashionable? Young people's perceptions of smoking in the media. Health Education Research 2003, 18:554-567.

50. Prokhorov AV, Winickoff JP, Ahluwalia JS, Ossip-Klein D, Tanski S, Lando HA, Moolchan ET, Muramoto M, Klein JD, Weitzman M, Ford KH, for the Tobacco Consortium AAoPCfCHR: Youth Tobacco Use: A Global Perspective for Child Health Care Clinicians. Pediatrics 2006, 118 e890-e903.

51. Sasco AJ, Secretan MB, Straif $K$ : Tobacco smoking and cancer: a brief review of recent epidemiological evidence. Lung Cancer 2004, 45:S3-S9.

52. Schulze A, Mons U: The evolution of educational inequalities in smoking: a changing relationship and a cross-over effect among German birth cohorts of 1921-70. Addiction 2006, 101:1051-1056.

53. Lavecchia C, Decarli A, Pagano R: Patterns of Smoking Initiation in Italian Males and Females from 1955 to 1985. Preventive Medicine 1995, 24:293-296.

54. Kessler RC, Angermeyer M, Anthony JC, De Graff R, Demyttenaere K, Gasquet I, et al: Lifetime prevalence and age-of-onset distributions of mental disorders in the World Health Organization's World Mental Health Survey Initiative. World Psychiatry 2007, 6:168-176.

55. Covey LS, Tam D: Depressive mood, the single-parent home, and adolescent cigarette smoking. American Journal of Public Health 1990, 80:1330-1333.

56. Escobedo LG, Reddy M, Giovino GA: The relationship between depressive symptoms and cigarette smoking in US adolescents. Addiction 1998, 93:433.

57. Massak A, Graham K: Is the smoking-depression relationship confounded by alcohol consumption? An analysis by gender. Nicotine \& Tobacco Research 2008, 10:1231-1243. 
58. Breslau N, Peterson EL, Schultz LR, Chilcoat HD, Andreski P: Major Depression and Stages of Smoking: A Longitudinal Investigation. Archives of General Psychiatry 1998, 55:161-166.

59. Pasco JA, Williams LJ, Jacka FN, Ng F, Henry MJ, Nicholson GC, Kotowicz MA, Berk M: Tobacco smoking as a risk factor for major depressive disorder: population-based study. The British Journal of Psychiatry 2008, 193:322-326.

60. Steuber TL, Danner F: Adolescent smoking and depression: Which comes first?. Addictive Behaviors 2006, 31:133-136.

61. Breslau N, Novak SP, Kessler RC: Psychiatric disorders and stages of smoking. Biological Psychiatry 2004, 55:69-76.

62. Siqueira L, Diab M, Bodian C, Rolnitzky L: Adolescents becoming smokers: the roles of stress and coping methods. Journal of Adolescent Health 2000, 27:399-408.

63. Kassel Jd, Stroud LR, Paronis CA: Smoking, stress, and negative affect: correlation, causation, and context across stages of smoking. Psychological Bulletin 2003, 129:270-304.

64. Anda RF, Croft JB, Felitti VJ, Nordenberg D, Giles WH, Williamson DF, Giovino GA: Adverse Childhood Experiences and Smoking During Adolescence and Adulthood. JAMA: The Journal of the American Medical Association 1999, 282:1652-1658.

65. Lloyd DA, Taylor J: Lifetime cumulative adversity, mental health and the risk of becoming a smoker. Health 2006, 10:95-112.

66. Schaap MM, Kunst AE, Leinsalu M, Regidor E, Ekholm O, Dzurova D, Helmert U, Klumbiene J, Santana P, Mackenbach JP: Effect of nationwide tobacco control policies on smoking cessation in high and low educated groups in 18 European countries. Tobacco Control 2008, 17:248-255.

67. Mullally BJ, Greiner BA, Allwright S, Paul G, Perry IJ: The effect of the Irish smoke-free workplace legislation on smoking among bar workers. Eur $J$ Public Health 2009, 19:206-211.

68. Fowkes FJl, Marlene CW, Stewart F, Gerald RFowkes, Amanda Amos, Price JF: Scottish smoke-free legislation and trends in smoking cessation. Addiction 2008, 103:1888-1895.

Pre-publication history

The pre-publication history for this paper can be accessed here:http://www. biomedcentral.com/1471-2458/10/74/prepub

doi:10.1186/1471-2458-10-74

Cite this article as: Oh et al: Determinants of smoking initiation among women in five European countries: a cross-sectional survey. BMC Public Health 2010 10:74.

\section{Submit your next manuscript to BioMed Central and take full advantage of:}

- Convenient online submission

- Thorough peer review

- No space constraints or color figure charges

- Immediate publication on acceptance

- Inclusion in PubMed, CAS, Scopus and Google Scholar

- Research which is freely available for redistribution 